\title{
ARTICLE \\ Naltrexone modulates contextual processing in depression
}

\author{
J. Chen (D) ${ }^{1}$, A. Mizuno ${ }^{1}$, T. Lyew ${ }^{1}$, H. T. Karim¹, J. F. Karp ${ }^{1}$, A. Y. Dombrovski $\mathbb{D}^{1}$ and M. Peciña ${ }^{1}$
}

\begin{abstract}
Context, the information surrounding an experience, can significantly alter the meaning and the affective responses to events. Yet the biological mechanisms through which context modulate experiences are not entirely understood. Here, we hypothesized that the $\mu$-opioid system - extensively implicated in placebo effects, a clinical phenomenon thought to rely on contextual processingmodulates the effects of contextual information on emotional attributions in patients with depression. To test this hypothesis, 20 unmedicated patients with depression completed a randomized, double-blind, placebo-controlled, crossover study of one dose of $50 \mathrm{mg}$ of naltrexone, or placebo immediately before completing two sessions of the Contextual Framing fMRI task. This task captures effects of valenced contextual cues (pleasant vs. unpleasant) on emotional attribution (the rating of subtle emotional faces: fearful, neutral, or happy). Behaviorally, we found that emotional attribution was significantly moderated by the interaction between contextual cues and subtle emotional faces, such that participants' ratings of valenced faces (fearful and happy), compared to neutral, were more negative during unpleasant, compared to pleasant context cues. At a neural level, context-induced blood-oxygen-level-dependent responses in the ventromedial prefrontal cortex, the dorsal anterior cingulate, the dorsolateral prefrontal cortex, and the lateral orbitofrontal cortex, significantly moderated the effects of context on emotional attribution, and were blunted by naltrexone. Furthermore, the effects of naltrexone on emotional attribution were partially abolished in more severely depressed patients. Our results provide insights into the molecular alterations underlying context representation in patients with depression, providing pivotal early data for future treatment studies.
\end{abstract}

Neuropsychopharmacology (2020) 45:2070-2078; https://doi.org/10.1038/s41386-020-00809-2

\section{INTRODUCTION}

Since the Kuleshov effect-or the altering of the meaning of an event by its pairing with a contextual image-was first described over a century ago [1], studies have extensively investigated the contribution of contextual processing to perception [2], including language [3], visual perception [4, 5], emotion [6-8], and social cognition[9]. Functional neuroimaging studies in healthy individuals have revealed that context exerts dominant top-down control over perception [10-12], facilitating object identification by reducing the amount of perceptual evidence needed to match an object with its unique representation $[5,13,14]$. Overall, this research has demonstrated that the study of context is essential to understand the influence of the real-world experiences on perception. Yet, the biological mechanisms of contextual effects are not entirely understood.

One example of contextual effects is the placebo response-the clinical improvement associated with inert treatments. In placebo research, expectations of improvement are often manipulated through the pairing of contextual cues (e.g., placebo pill) with verbal instructions. Consistent with the notion that contextual cues exert dominant top-down modulation of perception, functional neuroimaging studies of placebo analgesia have revealed activations in brain regions involved in cognitive control, emotional processing and valuation, such as the dorsolateral prefrontal cortex (dIPFC), the lateral orbitofrontal cortex (IOFC), and the ventromedial (vmPFC) [15-20], in parallel with reductions in somatosensory processing areas [16, 21, 22].
At a molecular level, neuropharmacological studies of placebo analgesia and antidepressant placebo effects have comprehensively characterized the role of the $\mu$-opioid system in placebo responses using $\mu$-opioid antagonists [23-25] and examining in vivo $\mu$-opioid receptor binding [17, 26-28]. Interestingly, while $\mu$-opioid receptors attain their highest concentrations subcortically (e.g. thalamus, periaqueductal gray, nucleus accumbens, and amygdala), where they modulate stress [29], reward [30], and emotion [31], studies of placebo responses have reliably implicated prefrontal changes in $\mu$-opioid neurotransmission $[17,18,26]$, consistently with a broader role of the $\mu$-opioid system in the modulation of expectancies and contextual cues. However, whether the $\mu$-opioid system is involved in contextual processing broadly, beyond placebo effects, and specifically whether the $\mu$-opioid system modulates emotional attribution, remains to be tested. If demonstrated, these effects would be particularly relevant for clinical conditions where $\mu$-opioid dysregulation has been demonstrated, such as major depressive disorder (MDD) [32].

In this study, we aimed to demonstrate that-as in studies of placebo responses-the effects of context on emotional attributions are modulated by $\mu$-opioid activation in prefrontal regions involved in cognitive control, emotional processing, and valuation. Consistently, results from a compelling study aimed at examining the neural correlates of the Kuleshov effect on emotional attributions, revealed that both positive and negative contexts result in significantly different ratings of

${ }^{1}$ Department of Psychiatry, University of Pittsburgh, Pittsburgh, PA, USA

Correspondence: M. Peciña (pecinam@upmc.edu)

These authors contributed equally: J. Chen, A. Mizuno

Received: 6 April 2020 Revised: 9 July 2020 Accepted: 11 August 2020

Published online: 25 August 2020 

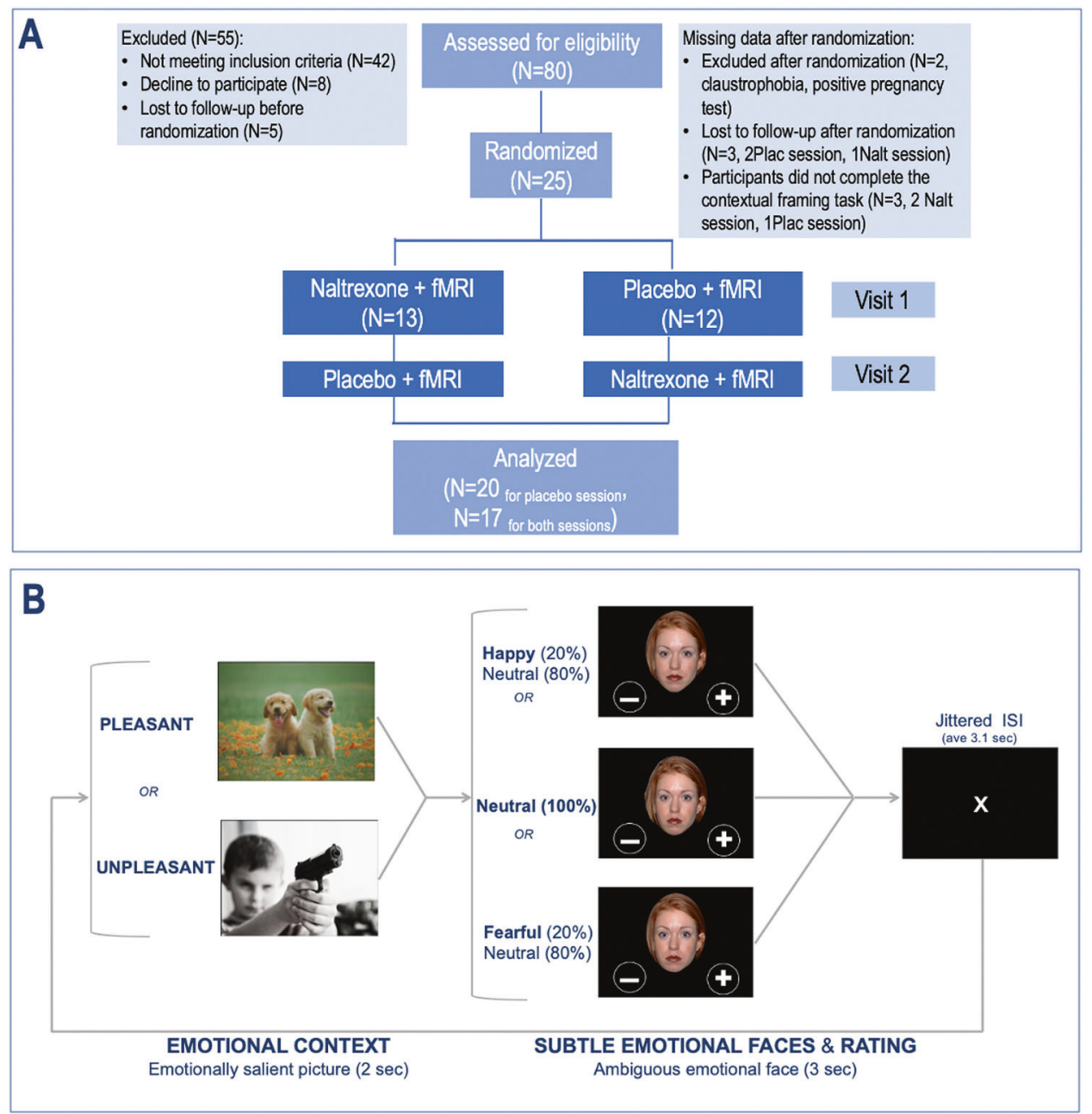

Fig. 1 Study design: participants completed a randomized, double-blind, placebo-controlled, crossover study of one dose of naltrexone $50 \mathbf{~ m g}$, or matching placebo. a, b Contextual framing fMRI task: Participants were first presented with an emotionally salient contextual image (from IAPS) for $2 \mathrm{~s}$ (context phase), followed by an ambiguous face to be rated as either positive or negative in $3 \mathrm{~s}$ (emotion and rating phase). Note: the examples of contextual images were replaced with similar copyright-free images from the website in order to comply with the IAPS usage agreement.

subtle emotional faces, compared with those presented under neutral contexts. At a neural level, contextual effects were found in inferotemporal and prefrontal regions-such as the vmPFC and the IOFC/ventrolateral PFC [33]. Here, 20 unmedicated patients with MDD completed two sessions of the Contextual Framing fMRI task, immediately after the administration of $50 \mathrm{mg}$ naltrexone-a potent $\mu$-opioid receptor antagonist, although not exclusively [34]—or matching placebo, in counterbalanced fashion, using a crossover, double-blind, repeated-measures design. This task aims to define the neural correlates of emotional contextual effects (pleasant vs. unpleasant images) during the rating of subtle emotional faces (happy/fearful/neutral). We hypothesized that the effects of valenced contextual cues on emotional attribution would be partly explained by changes in $\mu$-opioid neurotransmission in prefrontal regions involved in cognitive control, emotional processing and valuation, such as the dIPFC, the lateral OFC, and the VmPFC, and therefore blunted by the $\mu$-opioidantagonist naltrexone. Furthermore, $\mu$-opioid-dependent contextual effects will be reduced in more severely depressed individuals, as would be expected from reduced hedonic responses and lower levels of $\mu$-opioid receptor availability in depressed individuals [32].

\section{METHODS}

Participants and study design

We recruited 25 participants diagnosed with Major Depressive Disorder (MDD) through the University of Pittsburgh's recruitment website "Pitt+Me" (https://pittplusme.org) from July of 2017 to July of 2018. Enrolled participants were unmedicated, self-reported righthanded, and fluent in English, and provided written signed informed consent, approved by the University of Pittsburgh Institutional Review Board.

Using the Mini-International Neuropsychiatric Interview (M.I.N.I), we confirmed a diagnosis of non-psychotic MDD with or without anxiety disorders. Participants had Hamilton Depression Rating Scale (HDRS-17) score of $\geq 16$ at the screening visit, consistent with at least moderate depression, and were antidepressant medication-free for at least 21 days prior to collection of imaging data (5 weeks for fluoxetine). Exclusion criteria are described in the Supplementary Methods.

Participants underwent a randomized, double-blind, placebocontrolled, crossover study of one dose of oral $50 \mathrm{mg}$ naltrexone hydrochloride (ReVia ${ }^{\circledR}$ [package insert]. Toronto, ON: Teva Canada Limited; 2015) (onset of action: $\geq 15 \mathrm{~min}$; peak effect: $\sim 1 \mathrm{~h}$; duration: $\sim 24 \mathrm{~h}$ ) or matching placebo (Fig. 1a). The randomization list was generated by a group of independent pharmacists, who 
were unblinded to the study drug, while the participant and the study team were blinded. The randomization determined the order of the interventions (naltrexone or placebo, first or second visit). The two visits occurred within 7-10 days from one another. Naltrexone or placebo pills were ingested 60 min before the fMRI scanning session. Participants were informed that the purpose of the study was to investigate the effects of opioid blockade on emotional processing. They were also informed about naltrexone, including its pharmacological properties, its general clinical use, and its possible side effects (Supplementary Results). However, they were not informed about its potential effects on contextual processing.

Of the 25 enrolled participants who received the naltrexone/ placebo pill, two dropped out prior to scanning-one who was claustrophobic and another who had a positive pregnancy test. We excluded three additional participants who did not complete their placebo sessions. The final sample size was 20 for the placebo session, and 17 for the naltrexone session (three participants did not complete the fMRI session naltrexone session, but their results from their placebo session were still included in the analysis).

Clinical assessments

We assessed depressive symptoms using the Hamilton Depression Rating Scale (HDRS-17) [35], the Montgomery-Åsberg Depression Rating Scale (MADRS) [36], and the self-reported Quick Inventory of Depressive Symptomatology [37]. The HDRS was used to confirm eligibility based on depression severity and as the primary outcome measure. The MADRS was used to assess changes in depression severity between sessions.

\section{Contextual framing fMRI task}

The contextual framing task (Fig. $1 \mathrm{~b}$ ) was iteratively refined as a simpler version of a similar task utilized by Mobbs and colleagues [33]. In this mixed block/event-related design, participants saw an emotionally evocative/salient contextual image for $2 \mathrm{~s}$ (context phase) followed by the rating of the valence of an ambiguous emotional expression for $3 \mathrm{~s}$ (emotion and rating). The jittered inter-trial interval was $\sim 3 \mathrm{~s}(2-6 \mathrm{~s}$ range, S.D. $=1.1)$.

For the contextual images, we used a total of 72 emotionally evocative color photographs (36 pleasant and unpleasant images) from the International Affective Picture System (IAPS) database [38], which contains normalized scores of valences (pleasant $=9$, unpleasant $=1$ ) and arousal (high $=9$, low $=1$ ) for all the images. Based on these scores, we selected 72 contextual images with high or low valence scores (pleasant $>7.3$ and unpleasant $<3.4$ ) and moderate arousal score $(<6)$ to avoid excessively salient stimuli. This normalized image set provided the image-context for influencing the rating of ambiguous faces. We then generated three types of emotional faces with ambiguous expressions from the NIM-STIM database (http://www.macbrain.org/resources.htm) using a face-morphing software (FantaMorph 2.5). To validate the effectiveness of subtle face emotion without contextual effect, patients were asked to rate all task faces without a contextual image prior to entering the scanner (Supplementary Fig. 1).

Prior to the fMRI scan, participants received the following instructions: "You have two choices for rating each face: + (positive), indicating that the person seems happy, pleased, or delighted; or - (negative), indicating that the person seems sad, afraid, angry, or disgusted. You will see faces only for a brief moment, so please respond as fast as possible. The pictures of faces will alternate with other kinds of pictures." To avoid participants' guessing the premise of the task, we added the following instruction: "Pay attention to these non-face pictures because you'll be asked about them afterward. But remember that your main task is to rate the facial expressions." Participants reported their responses by clicking either the left or right index finger one of the buttons of the 5-button response box in each hand. They were instructed to rate the facial expression by pressing a right index finger to select positive $("+$ ") or a left index figure to select negative ("-") during the first run and the opposite during the second run.

Participants completed 144 trials (72 trials with each unique context picture, repeated twice) for each visit (Placebo and Naltrexone session). The task lasted $\sim 20 \mathrm{~min}$. Three types of ambiguous faces appeared equal times, and the sex of the face was randomized. The trial orders were optimized by using the easy-optimize-x tool that was implemented in MATLAB [39].

MRI data acquisition and preprocessing

MRI data were acquired according to standard procedures (Supplementary Methods). Acquired images were preprocessed using functions in the following software packages: NiPy [40], AFNI [41], BrainWavelet Toolbox [42], and the fMRI software library (FSL [43]). We have previously detailed this preprocessing pipeline elsewhere [44]. Specific methods and programs used were outlined in the Supplementary Methods.

\section{Statistical analysis for behavioral data}

We estimated effects of task conditions, depression severity-as measured by the HDRS - and extracted brain coefficients in hierarchical linear mixed-effects (LME) regression using the Ime4 package in R [45]. Models were fit using Restricted Maximum Likelihood (REML). Moderation effects were tested using higherorder interactions, and non-significant interactions were excluded from the models. To diagnose multicollinearity among predictors, we calculated variance inflation factors (VIFs). We ensured that predictors in all models met a rigorous criterion, VIF $<2$. Subject and run (clustering within-subject) intercepts were taken to be random in all models, and sex of faces was included as a nuisance fixed effect.

\section{MRI analysis}

MRI first level analysis. We constructed regressors for emotion and context type, and their interaction terms. The emotion regressor was coded as 1, for happy faces, 0 for neutral faces, and -1 for fearful faces. Similarly, the context regressor was coded as 1 for pleasant and -1 for unpleasant context. The interaction term included two regressors, one for the effect of context on happy faces ( 1 for pleasant context/happy faces, -1 for unpleasant context/happy faces, 0 for the remaining conditions), and another one for the effect of context on fearful faces ( 1 for pleasant context/fearful faces, -1 for unpleasant context/fearful faces, 0 for the remaining condition). We also included a trial regressor of no interest.

We aligned the boxcar regressors with trial onset (length $=$ trial duration) and convolved then with the hemodynamic response function. We then used the resulting time-series to regress against blood-oxygen-level-dependent (BOLD) signal for each run and participant, and constructed contrasts for each regressor (i.e., emotion, context type, emotion $\times$ context-type interaction).

Group level statistical analysis. We conducted group level voxelwise analyses using randomize in FSL. We used threshold-free cluster enhancement to determine significant clusters $(1-p>$ 0.05). For each contrast (emotion, context, emotion*context), we conducted one sample t-tests during the placebo session only. We then conducted paired samples t-test for each contrast (placebo > naltrexone and naltrexone $>$ placebo).

Neural contextual effects on ratings. We further investigated the relationship between neural contextual effects on in-scanner ratings (placebo session only). First, to ensure the extracted coefficients have anatomical significance, we apply the automated anatomical labeling (AAL) atlas [46] over the clusters that showed significant contextual effects (pleasant $>$ unpleasant) and created 


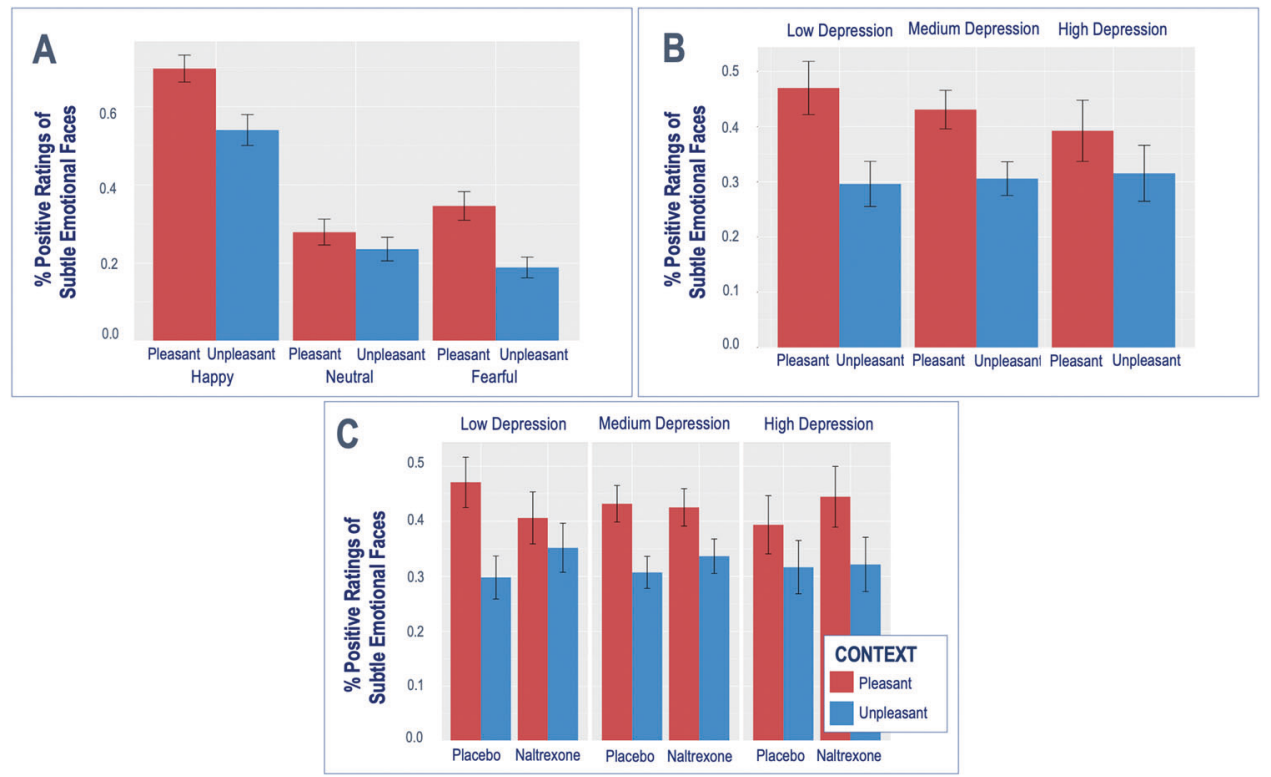

Fig. 2 Contextual effects on emotional attribution. Behavioral contextual effects (a) are moderated by depression severity (b) and naltrexone (c).

regions of interests that were both functionally and anatomically significant (see Supplementary Fig. 2 for the complete map). To examine the effect of BOLD responses on rating, we conducted the additional LME models, where mean coefficients from the regions of interests were extracted and included as fixed effects.

\section{RESULTS}

Context moderates the emotional attribution of subtle emotional faces

The final sample included 20 unmedicated participants with MDD (mean age $=24.0 \pm 0.2,80 \%$ female) with at least moderate depression (HDRS $\geq 16$ at screening, mean $=20.72 \pm 3.0$ ). Results regarding the effectiveness of our randomization procedures are reported in the Supplementary Results.

We first examined the effects of the task conditions [emotional context (pleasant/unpleasant) and subtle emotional faces (happy/ fearful/neutral)] on participant ratings using mixed-effects models (Fig. 2a). We found a significant context by emotion interaction, such that participants' ratings of valenced faces (happy and fearful), compared to neutral, were more negative during unpleasant, compared to the pleasant cues (Fig. 2a, happy: $b=$ $-0.54, \mathrm{SD}=0.15, Z=-3.15, p<0.001$; fearful: $b=-0.69, \mathrm{SD}=$ $0.15, Z=-4.34, p<0.001)$.

Depression severity moderates the effects of emotional context on facial emotion attribution

Contextual effects were further moderated by depression severity, such that the effect of context on participants' ratings was reduced in more severely depressed participants, as reflected by a depression severity by context interaction (Fig. $2 \mathrm{~b}, b=0.20$, SD $=$ $0.10, Z=2.06, p=0.040$ ).

Neural contextual effects

Whole-brain analyses revealed a significant effect of emotional context (pleasant $>$ unpleasant) on prefrontal responses in the vmPFC, the dorsomedial PFC/dorsal anterior cingulate cortex (medial: dmPFC/dACC), and the bilateral dIPFC extending into the IOFC, as well as in the caudate, inferior parietal and middle temporal gyrus and visual processing areas (Table 1A, top and Fig. 3a). No significant effects were seen for the opposite contrast (unpleasant $>$ pleasant).
BOLD responses during the processing of contextual information in these regions were not significantly correlated with depression severity (for all regions, $R<0.2, p>0.05$ ).

To understand the behavioral relevance of context-induced prefrontal responses to emotional attribution, we entered mean coefficients from the significant clusters during the processing of contextual cues (pleasant > unpleasant) into separate LME models predicting participants' ratings along with the task conditions. Greater vmPFC BOLD responses during context processing were associated with a reduced effect of context on participants' ratings, as reflected by a significant $\mathrm{vmPFC}^{*}$ context interaction (Fig. $3 \mathrm{~b}, b=0.27, \mathrm{SD}=0.11, Z=2.4, p=0.015$ ). In contrast, greater bilateral IOFC and dIPFC BOLD responses were associated with an increased effect of subtle emotional faces on positive emotional attribution (left IOFC: $b=0.24, \mathrm{SD}=0.11, Z=2.19, p=0.028$, right IOFC: $b=0.30, \mathrm{SD}=0.11, Z=2.75, p=0.006$; left dIPFC: $b=0.35$, $\mathrm{SD}=0.11, Z=3.15, p=0.002$, right dIPFC: $b=0.30, \mathrm{SD}=0.11$, $Z=2.65, p=0.008)$.

Naltrexone modulation of the effects of emotional context on facial emotion attribution

Then we examined the effects of a single dose of naltrexone, compared to placebo, on participants' ratings during the contextual framing task, as well as its interactions with depression severity. We found that naltrexone partially abolished the contextual effects on participants' ratings, but only in less severely depressed individuals, as reflected by a drug ${ }^{*}$ context ${ }^{*} H D R S$ interaction (Fig. $2 c, b=-0.35$, $\mathrm{SD}=0.15, Z=-2.34, p=0.019$ ).

Naltrexone modulation of neural contextual effects

At a neural level, the administration of a single dose of naltrexone blunted prefrontal, temporal, and occipital BOLD responses to the pleasant vs. unpleasant context with additional reductions in the brainstem (Fig. 4a and Table 1B). No effects were found for the opposite contrast.

Furthermore, mean coefficients from the contextual processing map were then entered into two separate LME models to predict ratings along with the drug and task conditions. In this case, lateral prefrontal regions that had shown an interaction with subtle emotional faces for the prediction of emotional attribution were combined into one single cluster (bilateral IOFC plus dIPFC), whereas the vmPFC that had shown an interaction with context for the 
Table 1. (A) Contextual framing task effects (pleasant > unpleasant). The voxel-wise neuroimaging analyses revealed a significant context effect (pleasant > unpleasant) in the vmPFC, bilateral OFC, dACC, and bilateral dIPFC, as well as in visual processing regions. (B) Naltrexone-induced changes during the contextual framing task. The administration of one single dose of the $\mu$-opioid-antagonist naltrexone was associated with significant decreases in BOLD responses during contextual processing (pleasant > unpleasant) in the medial PFC (vmPFC and dACC) and the bilateral OFC.

\begin{tabular}{|c|c|c|c|c|c|c|}
\hline \multicolumn{7}{|c|}{ A. One sample $t$-test: pleasant $>$ unpleasant $[\mathrm{PBO}]^{\mathrm{a}}$} \\
\hline 4598 & $\mathrm{~L} / \mathrm{R}$ & vmPFC (BA 32) and lateral OFC (BA 10) & 7.4 & -10 & 44 & -10 \\
\hline 655 & Bilateral & Superior occipital/cuneus/precuneus (BA 18/19/7) & 5.8 & 2 & -82 & 24 \\
\hline 536 & Right & Dorsolateral prefrontal [dIPFC (BA 8)] & 5.2 & 30 & 24 & 54 \\
\hline 133 & Left & Caudate & 4.4 & -12 & 18 & 10 \\
\hline 110 & Right & Inferior parietal (BA 40) & 4.7 & 50 & -48 & 52 \\
\hline 52 & Left & Middle temporal gyrus (BA 21) & 5.9 & -62 & -32 & -14 \\
\hline \multicolumn{7}{|c|}{ B. One sample $t$-test: pleasant $>$ unpleasant $[\mathrm{PBO}>\mathrm{NALT}]^{\mathrm{a}}$} \\
\hline 8598 & $\mathrm{~L} / \mathrm{R}$ & vmPFC (BA 32), lateral OFC (BA 10), dACC/dmPFC, dIPFC (BA 46, 8) & 8.1 & -8 & 42 & -8 \\
\hline 366 & Right & Middle temporal gyrus (BA 21) & 5.2 & 66 & -34 & -6 \\
\hline 325 & Left & Lingual gyrus (BA 30) & 4.6 & -12 & -42 & -6 \\
\hline 289 & Right & Brainstem & 5.6 & 10 & -26 & -20 \\
\hline 172 & Right & Precentral (BA 4) & 5.5 & 22 & -32 & 58 \\
\hline 93 & Left & Cerebellum & 4.8 & -32 & -80 & -42 \\
\hline
\end{tabular}

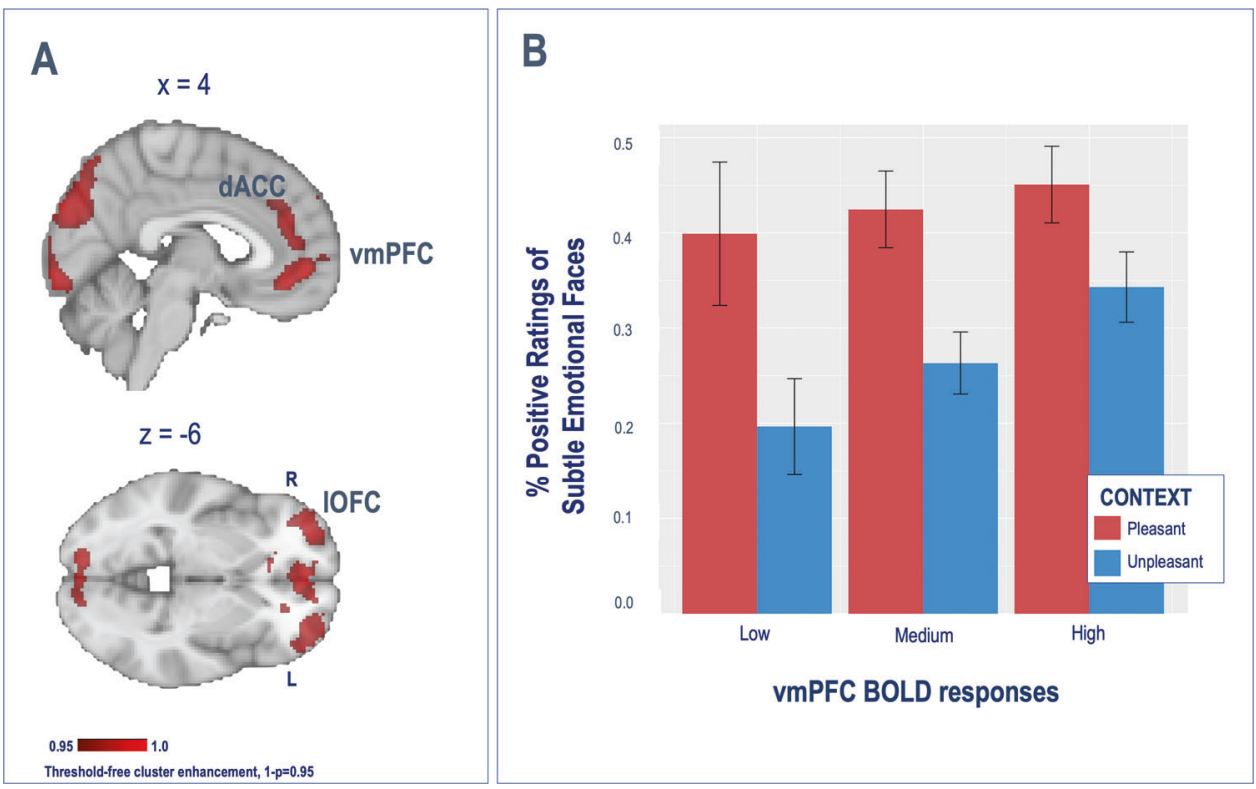

Fig. 3 Neural contextual effects. a Neural contextual framing neural effects (pleasant $>$ unpleasant). b Behavioral contextual effects are moderated by vmPFC BOLD responses.

prediction of emotional attribution was kept as a single cluster. Results from this model revealed that the effects of context on emotional attribution were moderated by the two independent variables, brain responses (vmPFC or IOFC/dIPFC) and drug type (placebo vs. naltrexone), as reflected by a significant three-way interaction in each model.
Compare to the placebo session, naltrexone diminished the vmPFC moderation of the effect of context on emotional attribution (Fig. 4b, $b=-0.37, \mathrm{SD}=0.16, Z=-2.3, p=0.02$ ), while it enhanced the IOFC/dIPFC moderation of the effect of context on emotional attribution (Fig. $4 c, b=-0.36, \mathrm{SD}=0.14$, $Z=-2.15, p=0.03$ ) 

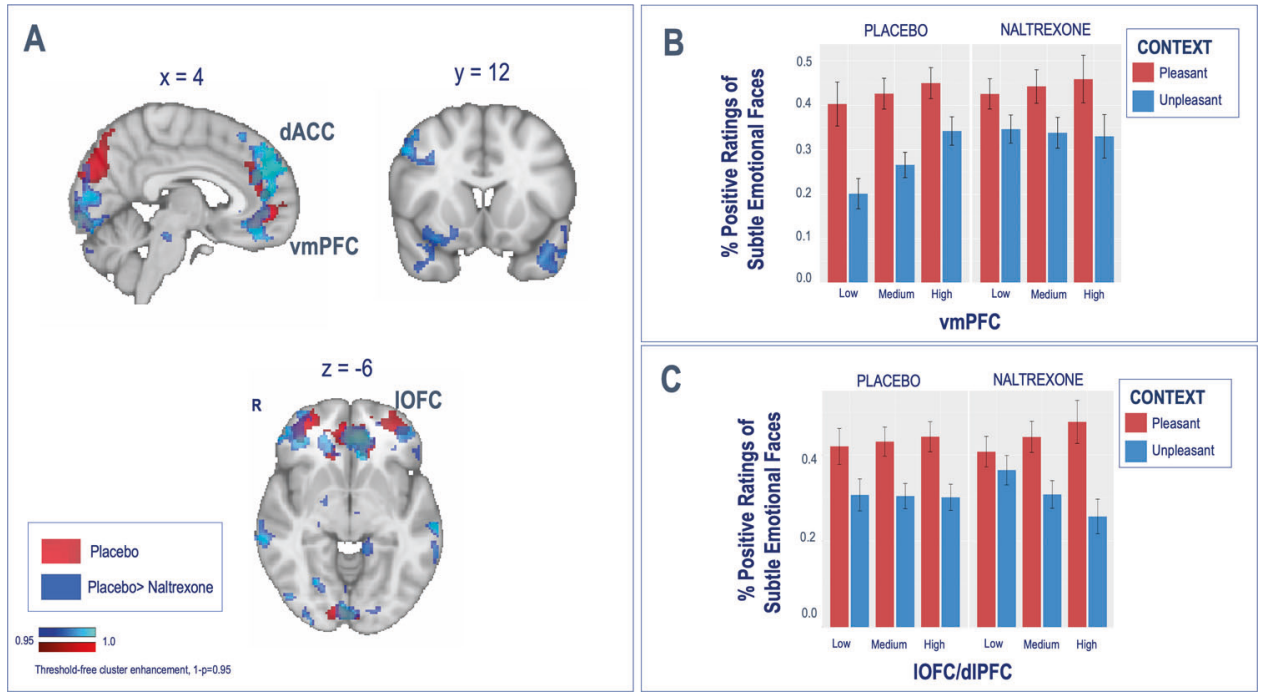

Fig. 4 Naltrexone modulation of neural contextual effects. a Naltrexone-induced modulation of contextual effects (pleasant $>$ unpleasant) during the contextual framing task: the administration of one single dose of naltrexone was associated with significant decreases in BOLD responses in the vmPFC and the bilateral IOFC. $\mathbf{b}$, c Naltrexone differentially reduced the effect of context on emotional attribution in participants with reduced VmPFC responses, whereas it enhanced the effect of context in emotional attribution in participants with high IOFC/dIPFC responses.

\section{DISCUSSION}

Our results support the significant role of naltrexone on contextual processing on emotional attributions in patients with MDD. Specifically, we found that emotional attribution was significantly moderated by the interaction between contextual cues and subtle emotional faces, such that participants' ratings of valenced faces, compared to neutral, were more negative during unpleasant, compared to the pleasant cues. At a neural level, context-induced BOLD responses in the VmPFC, dACC, dIFPC, and IOFC, were blunted by naltrexone. In addition, naltrexone effects on contextinduced BOLD responses significantly moderated the effects of context on emotional attribution. Furthermore, the effects of naltrexone on emotional attribution were only partially abolished in more severely depressed patients.

Broadly, contextual cues-or the information surrounding emotional faces-can include within-face [47] or -body features (e.g., posture) $[48,49]$, but also external cues, such as emotional labels, verbal descriptions, or visual scenes $[8,33]$, like those used in this study. Contextual frames are thought to be integrated into social attributions through expectations where, based on experience, the attributor's judgments can be shifted from one category to another [50]. The context might indeed facilitate the retrieval of information [14, 51, 52], whereby top-down contextual information and bottom-up perception [5], can activate associated information within the context frame, increasing the expectancy for specific stimuli [50]. As described by Mobbs and colleagues [33], we found that emotional attribution was significantly moderated by the interaction between contextual cues and subtle emotional faces, such that participants' ratings of valenced faces were significantly influenced by context. Surprisingly, we did not observe a significant contextual effect during the processing of more ambiguous neutral faces. This might be explained by reduced contextual effects during the processing of incongruent information since our experiment lacked a neutral context condition. Consistently, studies examining contextual effects on perception have shown worse performance under incongruent conditions [53]. Under these circumstances, participants might guide their responses based on personal biases rather than contextual effects.

Our imaging results further demonstrate the role of the prefrontal cortex in contextual processing. Indeed, we found that pleasant contexts, compared to unpleasant, were associated with increased $B O L D$ responses extensively in cortical regions, such as the vmPFC, the dACC, and the bilateral dIPFC and IOFC, as well as in the caudate, inferior parietal and middle temporal gyrus, and visual processing areas. Among the areas of activation, only vmPFC BOLD responses interacted with context to predict emotional attribution, such that the effect of context on emotional attribution was larger in participants with reduced vmPFC BOLD responses. The vmPFC subserves value-based decision making [54], emotion regulation, and the processing of self-relevant information [55]. Furthermore, it has been argued that the vmPFC is critical when affective responses are shaped by conceptual information, providing meaning to a situation by selectively integrating relevant contextual and internal cues [56]. Furthermore, the vmPFC has been robustly involved in placebo analgesia $[15,57,58]$ and antidepressant placebo effects $[28,59]$.

In addition, we found that context-induced bilateral IOFC/dIPFC $B O L D$ responses further moderated emotional attribution through its interaction with subtle emotional faces. In this case, patients with greater context-induced IOFC/dIPFC BOLD responses showed a greater effect of face type on positive emotional attribution. Consistently, studies of emotional reappraisal (e.g., using contextual images during the presentation of negative emotions) have demonstrated that increased brain responses in the IOFC/ VIPFC and dIPFC during reappraisal are associated with reduced negative feelings in response to negative emotions [60, 61]. Altogether, our results support that-rather than an effect of the VmPFC and vIPFC in positive and negative attribution respectively, as described by Mobbs et al. $[33,62]$ - the vmPFC might have a key role implicitly integrating the meaning of relevant contextual cues. In contrast, the lateral prefrontal cortex might be involved in bringing emotional representations on-line and inhibiting negative affect during the processing of contextual cues, as suggested by current theories of emotion regulation $[63,64]$.

As hypothesized, these effects were modulated by naltrexone. We demonstrated that one dose of the $\mu$-opioid-antagonist naltrexone decreased context-induced BOLD responses extensively in the $\mathrm{vmPFC/dACC}$ and the bilateral dIPFC/IOFC. Interestingly, behaviorally, naltrexone differentially reduced the vmPFC- and enhanced the IOFC/dIPFC-dependent effect of context on emotional 
attribution, suggesting that under naltrexone, contextual effects might relay predominantly in lateral and dorsal prefrontal regions. $\mu$-Opioid receptors are primarily known for their role in nociception and analgesic effects $[17,65]$ and the regulation of naturally important stimuli (e.g., appetite, reproduction) and reinforcement [66]. $\mu$-Opioid receptors are also centrally involved in responses to social stimuli, whether modulating the distress of social rejection or the positive responses to social acceptance and affiliative behavior $[67,68]$. The role of $\mu$-opioid receptors in cognition is much less well understood, although emerging evidence points to a significant role in decision making and cognitive control [69]. Of relevance to our results here, prefrontal $\mu$-opioid activation has extensively been demonstrated in the modulation of expectancies-often developed by contextual cues-in placebo analgesia experiments $[18,27,70]$. In addition, animal studies have demonstrated the role of prefrontal structures in hedonic coding [71]. For example, vmPFC microinjections of a $\mu$-opioid agonist increases eating behavior and intake of food [72], and OFC electrical stimulation has shown to promote self-stimulation behavior [73] in rats. These results support the hypothesis that $\mu$-opioid receptors in prefrontal regions might encode value representation of context and the associated hedonic responses. This hypothesis is further supported by the fact that we only observed the blunting effect of naltrexone during the processing of positively valenced cues.

In addition to Naltrexone's well-known antagonist effect on $\mu$ opioid and other opioid receptors, $(+)$-naloxone and $(+)$-naltrexone, inactive enantiomers have shown antagonist effects at the toll-like receptor 4 (TLR4) site in vitro and in vivo [74, 75]. This mechanism of action is responsible for the anti-inflammatory properties of low-dose-naltrexone [76] but has failed to block the neurochemical or behavioral abuse-related effects of cocaine and opioid agonist using a wide range of does and experimental conditions [77], contrary to initial results [78]. In addition, while little is known regarding the role of TLRs in cognition, some evidence indicates a developmental role in shaping spatial reference memory, and fear learning and memory. Although TLR4 antagonist showed no effect regulating spatial and contextual hippocampus-dependent cognitive behavior [79]. Still, it is plausible to believe that the effects observed here could be explained by a TLR4-antagonism hypothesis, which could be the focus of future investigations.

The results described above were further moderated by depression severity. In particular, more severely depressed individuals had reduced contextual effects. Consistently, studies of mood indicate that positive affect evokes the generation of more unique associations in free-association tasks [80], promoting more creative problem solving [81], and that depressive features, such as ruminative thinking, limit the associability between context and perception [82]. Studies in MDD have further demonstrated deficits in contextual processing when retaining contextual information, such as increased context-dependent errors, compared to healthy controls [83], especially when interfering with negative stimuli [84]. Interestingly, we found that one single dose of naltrexone significantly reduced the effects of context on facial emotion attributions in less severely depressed participants. These results are in line with the prominent role of $\mu$-opioid receptors in depression [32], and a down-regulation of $\mu$-opioid receptors in more severely depressed patients [28]. Alternatively, these results are also consistent with the role of TLR4 in depression. Preclinical studies have shown that TLR4 expression in the prefrontal cortex is enhanced in a stress-based model of depression [85], and that altered peripheral expression of TLRs appears to be associated with depression and may account for the heightened inflammatory state [86]. Furthermore, some antidepressant and anti-inflammatory agents are able to attenuate the depressive-like behavior induced by lipopolysaccharide, a TLR4 agonist [87].

Our study has several limitations. First, our experimental design did not include a neutral context condition, as in previous studies
[33]. This decision was based on our initial hypothesis regarding $\mu$-opioid-dependent positive valenced contextual effects. As such, we expected to have greater power to detect significant effects when comparing pleasant to unpleasant context. Furthermore, Mobbs and colleagues found no significant differences in contextinduced brain responses between positive and negative context, compared to neutral, which also pointed to the lack of need of a neutral context. Our study has a modest sample size and lacks a control group. Future studies should compare our findings to nonclinical and other clinical populations in larger samples. Finally, because $82 \%$ of female participants were using hormonal birth control, which is known to affect hormonal test results [88], we did not control for hormone levels (e.g., estradiol), which have been previously linked to opioid receptor availability [89] and TLR gene expression [90]. Therefore, individual differences in female subjects' menstrual day cycle could have affected our results.

Overall, our results support the naltrexone blockade of prefrontal signal modulating contextual effects on emotional attribution in patients with MDD. These findings might well pertain to psychiatric conditions beyond mood disorders, where contextual deficits lead to a wide variety of symptoms-rom paranoid beliefs or intrusive thoughts to compulsive behaviorsseen in multiple psychiatric disorders, including schizophrenia [91], autism [92] or post-traumatic stress disorder [93]. Still, our understanding of the treatments that might help remediate these deficits is lacking. Our findings shed light on the molecular understanding of contextual effects in patients with depression, opening the possibility of pharmacological treatments for the treatment of contextual processing deficits in psychiatric conditions. A naltrexone-based therapy targeting hedonic responses to contextual cues, while ensuring low or absent addictive potential, would be expected to confer clinical benefits that are distinct from monoamine-based therapies, particularly in patients who are inadequately responsive to standard antidepressants [32]. Furthermore, this targeted approach to drug development might help improve results from on-going trials of very doses of $\mu$ opioid agonists, and $\mu$-opioid agonist-antagonist combinations, which have failed to demonstrate sufficient evidence of effectiveness for the treatment of MDD [94]. Finally, naltrexone could potentially be used to reduce placebo effects in clinical trials and improve assay sensitivity.

\section{FUNDING AND DISCLOSURE}

This work was supported by the K23 MH108674 (MP) and the Brain \& Behavior Research Foundation Young Investigator Award 23730 (MP). The funders had no role in the design and conduct of the study; collection, management, analysis, and interpretation of the data; preparation, review, or approval of the manuscript; and decision to submit the manuscript for publication. JFK received medication supplies from Indivior and Pfizer for investigator-initiated studies, received an honorarium from Otsuka for disease-specific educational activity, and receives compensation for editorial work for the American Association of Geriatric Psychiatry and Journal of Clinical Psychiatry. The other authors have no interests to disclose that are or might be perceived to be in conflict with the work reported in this study. Clinical trial number: NCT04322526. The authors declare no competing interests.

\section{AUTHOR CONTRIBUTIONS}

Author's contributions are stated below: substantial contributions to the conception or design of the work (AM, HK, MP) or the acquisition (TL, JFK, AD, MP), analysis (JC, $A M, H K, M P)$, or interpretation of data (JC, AM, HK, AD, MP), and drafting or revising it critically for important intellectual content (JC, AM, TL, HK, JFK, AD, MP). Final approval of the version to be published; and agreement to be accountable for all aspects of the work in ensuring that questions related to the accuracy or integrity of any part of the work are appropriately investigated and resolved (JC, AM, MP) 


\section{ADDITIONAL INFORMATION}

Supplementary Information accompanies this paper at (https://doi.org/10.1038/ s41386-020-00809-2).

Publisher's note Springer Nature remains neutral with regard to jurisdictional claims in published maps and institutional affiliations.

\section{REFERENCES}

1. Kawin BF. How movies work. London: University of California Press; 1992.

2. Chao, ZC, Nagasaka Y, Fujii, N. Cortical network architecture for context processing in primate brain. eLife. 2015;4:e06121. https://elifesciences.org/articles/ 06121. Accessed 11 Feb 2020.

3. Hagoort P. On Broca, brain, and binding: a new framework. Trends Cogn Sci. 2005;9:416-23.

4. Schwartz O, Hsu A, Dayan P. Space and time in visual context. Nat Rev Neurosci. 2007;8:522-35.

5. Bar M. Visual objects in context. Nat Rev Neurosci. 2004;5:617-29.

6. de Gelder B. Towards the neurobiology of emotional body language. Nat Rev Neurosci. 2006;7:242-9.

7. Barrett LF, Kensinger EA. Context is routinely encoded during emotion perception. Psychol Sci. 2010;21:595-9.

8. Barrett LF, Lindquist KA, Gendron M. Language as context for the perception of emotion. Trends Cogn Sci. 2007;11:327-32.

9. Levy LH. Context effects in social perception. J Abnorm Soc Psychol. 1960;61:295-7.

10. Cox D, Meyers E, Sinha P. Contextually evoked object-specific responses in human visual cortex. Science. 2004;304:115-7.

11. Maratos EJ, Dolan RJ, Morris JS, Henson RNA, Rugg MD. Neural activity associated with episodic memory for emotional context. Neuropsychologia. 2001;39:910-20.

12. Bar M, et al. Top-down facilitation of visual recognition. PNAS. 2006;103:449-54.

13. Bar M, Aminoff E. Cortical analysis of visual context. Neuron. 2003;38:347-58.

14. Bar M, Ullman S. Spatial context in recognition. Perception. 1996;25:343-52.

15. Petrovic P. Placebo and opioid analgesia- imaging a shared neuronal network. Science. 2002;295:1737-40.

16. Wager TD, et al. Placebo-induced changes in FMRI in the anticipation and experience of pain. Science. 2004;303:1162-7.

17. Zubieta J-K, et al. Placebo effects mediated by endogenous opioid activity on mu-opioid receptors. J Neurosci. 2005;25:7754-62.

18. Peciña M, Stohler CS, Zubieta J-K. Neurobiology of placebo effects: expectations or learning? Soc Cogn Affect Neurosci. 2014;9:1013-21.

19. Tracey I, Mantyh PW. The cerebral signature for pain perception and its modulation. Neuron. 2007;55:377-91.

20. Bingel U, Lorenz J, Schoell E, Weiller C, Büchel C. Mechanisms of placebo analgesia: rACC recruitment of a subcortical antinociceptive network. Pain. 2006;120:8-15.

21. Price DD, Craggs J, Verne GN, Perlstein WM, Robinson ME. Placebo analgesia is accompanied by large reductions in pain-related brain activity in irritable bowel syndrome patients. Pain. 2007;127:63-72.

22. Eippert $F$, et al. Activation of the opioidergic descending pain control system underlies placebo analgesia. Neuron. 2009;63:533-43.

23. Amanzio M, Benedetti F. Neuropharmacological dissection of placebo analgesia: expectation-activated opioid systems versus conditioning-activated specific subsystems. J Neurosci. 1999;19:484-94.

24. Benedetti $F$. The opposite effects of the opiate antagonist naloxone and the cholecystokinin antagonist proglumide on placebo analgesia. Pain. 1996;64:535-43.

25. Levine JD, Gordon NC, Fields HL. The mechanism of placebo analgesia. Lancet. 1978;2:654-7.

26. Wager TD, Scott DJ, Zubieta JK. Placebo effects on human mu-opioid activity during pain. Proc Natl Acad Sci USA. 2007;104:11056-61.

27. Pecina M, Zubieta J-K. Expectancy modulation of opioid neurotransmission. Int Rev Neurobiol. 2018;138:17-37.

28. Pecina $M$, et al. Association between placebo-activated neural systems and antidepressant responses: neurochemistry of placebo effects in major depression. JAMA Psychiatry. 2015;72:1087-94.

29. Emery MA, Akil H. Endogenous opioids at the intersection of opioid addiction, pain, and depression: the search for a precision medicine approach. Ann Rev Neurosci. 2020;3:355-74.

30. Pecina S, Berridge KC. Hedonic hot spot in nucleus accumbens shell: where do mu-opioids cause increased hedonic impact of sweetness? J Neurosci. 2005; 25:11777-86.

31. Kennedy SE, Koeppe RA, Young EA, Zubieta J-K. Dysregulation of endogenous opioid emotion regulation circuitry in major depression in women. Arch Gen Psychiatry. 2006;63:1199-208.
32. Pecina $M$, et al. Endogenous opioid system dysregulation in depression: implications for new therapeutic approaches. Mol Psychiatry. 2018;24:576-87.

33. Mobbs $D$, et al. The Kuleshov effect: the influence of contextual framing on emotional attributions. Soc Cogn Affect Neurosci. 2006;1:95-106.

34. Verebey K, Mulé SJ. Naltrexone pharmacology, pharmacokinetics, and metabolism: current status. Am J Drug Alcohol Abus. 1975;2:357-63.

35. Hamilton M. A rating scale for depression. J Neurol Neurosurg Psychiatry. 1960;23:56-62.

36. Montgomery SA, Asberg M. A new depression scale designed to be sensitive to change. Br J Psychiatry. 1979;134:382-9.

37. Rush AJ, Gullion CM, Basco MR, Jarrett RB, Trivedi MH. The Inventory of Depressive Symptomatology (IDS): psychometric properties. Psychol Med. 1996;26:477-86.

38. Lang PJ, Bradley, MM, Cuthbert, BN. International Affective Picture System (IAPS): technical manual and affective ratings. Gainesville: University of Florida, Center for Research in Psychophysiology; 1995.

39. Bob Spunt. easy-optimize-x: Formal Release for Archiving on Zenodo. Zenodo; 2016.

40. Gorgolewski K, et al. Nipype: a flexible, lightweight and extensible neuroimaging data processing framework in python. Front Neuroinformatics. 2011;5:13.

41. Cox RW. AFNI: software for analysis and visualization of functional magnetic resonance neuroimages. Comput Biomed Res. 1996;29:162-73.

42. Patel $A X$, et al. A wavelet method for modeling and despiking motion artifacts from resting-state fMRI time series. Neuroimage. 2014;95:287-304.

43. Jenkinson M, Beckmann CF, Behrens TEJ, Woolrich MW, Smith SM. FSL. Neurolmage. 2012;62:782-90.

44. Vanyukov PM, Hallquist MN, Delgado M, Szanto K, Dombrovski AY. Neurocomputational mechanisms of adaptive learning in social exchanges. Cogn Affect Behav Neurosci. 2019;19:985-97.

45. Bates D, Mächler M, Bolker B, Walker S. Fitting linear mixed-effects models using Ime4. J Stat Softw. 2015;67:1-48.

46. Tzourio-Mazoyer N, et al. Automated anatomical labeling of activations in SPM using a macroscopic anatomical parcellation of the MNI MRI single-subject brain Neurolmage. 2002;15:273-89.

47. Boll S, Gamer M, Kalisch R, Büchel C. Processing of facial expressions and their significance for the observer in subregions of the human amygdala. Neuroimage. 2011;56:299-306.

48. Aviezer $\mathrm{H}$, et al. Angry, disgusted, or afraid? Studies on the malleability of emotion perception. Psychol Sci. 2008. https://journals.sagepub.com/doi/full/ 10.1111/j.1467-9280.2008.02148.x?url_ver=Z39.88-2003\&rfr_id=ori\%3Arid\% 3Acrossref.org\&rfr_dat=cr_pub\%3Dpubmed. Accessed 12 Feb 2020

49. Meeren HKM, van Heijnsbergen CCRJ, de Gelder B. Rapid perceptual integration of facial expression and emotional body language. Proc Natl Acad Sci USA 2005;102:16518-23.

50. Trope Y. Identification and inferential processes in dispositional attribution. Psychol Rev. 1986;93:239-57.

51. Mandler J, Johnson N. Some of the thousand words a picture is worth. J Exp Psychol Hum Learn Mem. 1976;2:529-40.

52. Minsky M. A framework for representing knowledge. In: Winston, editor. The psychology of computer vision. London: Addison-Wesley Publishing Company; 1974.

53. Rémy $F$, et al. Object recognition in congruent and incongruent natural scenes: a life-span study. Vis Res. 2013;91:36-44.

54. Fellows LK, Farah MJ. Ventromedial frontal cortex mediates affective shifting in humans: evidence from a reversal learning paradigm. Brain. 2003;126:1830-7.

55. Hiser J, Koenigs $M$. The multifaceted role of the ventromedial prefrontal cortex in emotion, decision making, social cognition, and psychopathology. Biol Psychiatry. 2018;83:638-47.

56. Roy M, Shohamy D, Wager TD. Ventromedial prefrontal-subcortical systems and the generation of affective meaning. Trends Cogn Sci. 2012;16:147-56.

57. Tinnermann A, Geuter S, Sprenger C, Finsterbusch J, Büchel C. Interactions between brain and spinal cord mediate value effects in nocebo hyperalgesia. Science. 2017;358:105-8.

58. Geuter S, Koban L, Wager TD. The cognitive neuroscience of placebo effects: concepts, predictions, and physiology. Annu Rev Neurosci. 2017;40:167-88.

59. Sikora M, et al. Salience network functional connectivity predicts placebo effects in major depression. Biol Psychiatry Cogn Neurosci Neuroimaging. 2016;1:68-76.

60. Phan KL, et al. Neural substrates for voluntary suppression of negative affect: a functional magnetic resonance imaging study. Biol Psychiatry. 2005;57:210-9.

61. Ochsner $\mathrm{KN}$, et al. For better or for worse: neural systems supporting the cognitive down- and up-regulation of negative emotion. Neuroimage. 2004;23:483-99.

62. $\mathrm{Kim} \mathrm{H}$, et al. Contextual modulation of amygdala responsivity to surprised faces. J Cogn Neurosci. 2004;16:1730-45.

63. Phillips ML, Drevets WC, Rauch SL, Lane R. Neurobiology of emotion perception I: the neural basis of normal emotion perception. Biol Psychiatry. 2003;54:504-14.

64. Braunstein LM, Gross JJ, Ochsner KN. Explicit and implicit emotion regulation: a multi-level framework. Soc Cogn Affect Neurosci. 2017;12:1545-57. 
65. Zubieta JK, et al. Regional mu opioid receptor regulation of sensory and affective dimensions of pain. Science. 2001;293:311-5.

66. Shippenberg TS, Elmer GI. The neurobiology of opiate reinforcement. Crit Rev Neurobiol. 1998;12:267-303.

67. Hsu DT, et al. It still hurts: altered opioid activity in the brain during social rejection and acceptance in major depressive disorder. Mol Psychiatry. 2015;20:193-200.

68. Hsu DT, et al. Response of the $\mu$-opioid system to social rejection and acceptance. Mol Psychiatry. 2013;18:1211-7.

69. van Steenbergen $\mathrm{H}$, Eikemo $\mathrm{M}$, Leknes $\mathrm{S}$. The role of the opioid system in decision making and cognitive control: a review. Cogn Affect Behav Neurosci. 2019;19:435-58.

70. Pecina $M$, et al. Personality trait predictors of placebo analgesia and neurobiological correlates. Neuropsychopharmacology. 2013;38:639-46.

71. Castro DC, Berridge KC. Opioid and orexin hedonic hotspots in rat orbitofrontal cortex and insula. PNAS. 2017;114:E9125-34.

72. Mena JD, Sadeghian K, Baldo BA. Induction of hyperphagia and carbohydrate intake by $\mu$-opioid receptor stimulation in circumscribed regions of frontal cortex. J Neurosci. 2011;31:3249-60.

73. Routtenberg A, Sloan M. Self-stimulation in the frontal cortex of Rattus norvegicus. Behav Biol. 1972;7:567-72.

74. Lewis SS, et al. (+)-Naloxone, an opioid-inactive toll-like receptor 4 signaling inhibitor, reverses multiple models of chronic neuropathic pain in rats. J Pain. 2012;13:498-506.

75. Hutchinson MR, et al. Non-stereoselective reversal of neuropathic pain by naloxone and naltrexone. Eur J Neurosci. 2008;28:20-9.

76. Cant R, Dalgleish AG, Allen RL. Naltrexone inhibits IL-6 and TNFa production in human immune cell subsets following stimulation with ligands for intracellular toll-like receptors. Front Immunol. 2017;8:809.

77. Tanda $G$, et al. Lack of specific involvement of $(+)$-naloxone and $(+)$-naltrexone on the reinforcing and neurochemical effects of cocaine and opioids. Neuropsychopharmacology. 2016;41:2772-81.

78. Hutchinson MR, et al. Opioid activation of toll-like receptor 4 contributes to drug reinforcement. J Neurosci. 2012;32:11187-200.

79. Okun E, et al. Evidence for a developmental role for TLR4 in learning and memory. PLoS ONE. 2012;7:e47522. https://journals.plos.org/plosone/article?id=10.1371/ journal.pone.0047522. Accessed 6 July 2020.

80. Isen AM, Daubman KA. The influence of affect on categorization. J Pers Soc Psychol. 1984;47:1206-17.
81. Isen AM, Johnson MM, Mertz E, Robinson GF. The influence of positive affect on the unusualness of word associations. J Pers Soc Psychol. 1985;48:1413-26.

82. Harel EV, Tennyson RL, Fava M, Bar M. Linking major depression and the neural substrates of associative processing. Cogn Affect Behav Neurosci. 2016;16:1017-26.

83. Msetfi RM, Murphy RA, Kornbrot DE, Simpson J. Impaired context maintenance in mild to moderately depressed students. Q J Exp Psychol. 2009;62:653-62.

84. Masuyama A, Kaise Y, Sakano Y, Mochizuki S. The interference of negative emotional stimuli on context processing in mildly depressed undergraduates. Cogent Psychol. 2018;5:1424681.

85. Gárate I, et al. Stress-induced neuroinflammation: role of the Toll-like receptor-4 pathway. Biol Psychiatry. 2013;73:32-43.

86. Liu J, Buisman-Pijlman F, Hutchinson MR. Toll-like receptor 4: innate immune regulator of neuroimmune and neuroendocrine interactions in stress and major depressive disorder. Front Neurosci. 2014;8:309.

87. Salazar A, Gonzalez-Rivera BL, Redus L, Parrott JM, O'Connor JC. Indoleamine 2,3dioxygenase mediates anhedonia and anxiety-like behaviors caused by peripheral lipopolysaccharide immune challenge. Horm Behav. 2012;62:202-9. https://pubmed.ncbi.nlm.nih.gov/22504306/. Accessed 2 July 2020.

88. Weindling $\mathrm{H}$, Henry JB. Laboratory test results altered by 'The Pill'. JAMA. 1974;229:1762-68. https://jamanetwork.com/journals/jama/fullarticle/356999. Accessed 2 July 2020.

89. Smith YR, et al. Brain opioid receptor measurements by positron emission tomography in normal cycling women: relationship to luteinizing hormone pulsatility and gonadal steroid hormones. J Clin Endocrinol Metab. 1998;83: 4498-505.

90. Zandieh $Z$, et al. The effect of estradiol and progesterone on toll like receptor gene expression in a human fallopian tube epithelial cell line. Cell J. 2016;17: 678-91.

91. Champagne-Lavau M, Charest A. Theory of mind and context processing in schizophrenia: the role of social knowledge. Front Psychiatry. 2015;6:98.

92. Jasmin $\mathrm{K}$, et al. Overt social interaction and resting state in young adult males with autism: core and contextual neural features. Brain. 2019;142:808-22.

93. Liberzon I, Abelson JL. Context processing and the neurobiology of post-traumatic stress disorder. Neuron. 2016;92:14-30.

94. FierceBiotech. FDA rejects Alkermes' depression drug ALKS 5461. 2019. https:// www.fiercebiotech.com/biotech/fda-rejects-alkermes-depression-drug-alks-5461. Accessed 27 May 2020. 